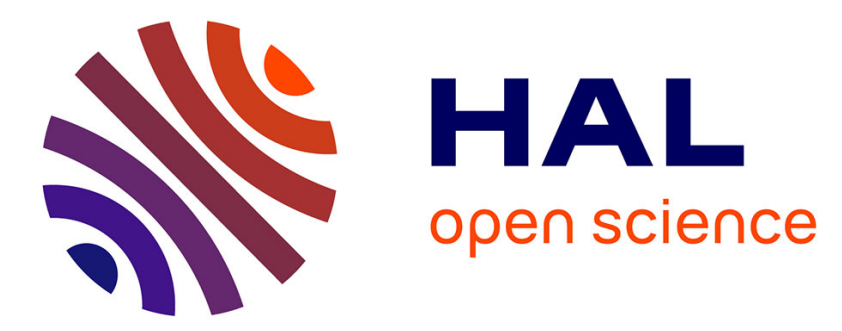

\title{
Le fourreau d'épée celtique décoré de Baron-sur-Odon (Calvados)
}

\author{
Dominique Bertin, Daniel Dufournier
}

\section{To cite this version:}

Dominique Bertin, Daniel Dufournier. Le fourreau d'épée celtique décoré de Baron-sur-Odon (Calvados). Gallia - Fouilles et monuments archéologiques en France métropolitaine, 1974, 32 (2), pp.243-248. 10.3406/galia.1974.2665. hal-01934631

\section{HAL Id: hal-01934631 \\ https://hal.science/hal-01934631}

Submitted on 25 Feb 2020

HAL is a multi-disciplinary open access archive for the deposit and dissemination of scientific research documents, whether they are published or not. The documents may come from teaching and research institutions in France or abroad, or from public or private research centers.
L'archive ouverte pluridisciplinaire HAL, est destinée au dépôt et à la diffusion de documents scientifiques de niveau recherche, publiés ou non, émanant des établissements d'enseignement et de recherche français ou étrangers, des laboratoires publics ou privés.

\section{(이) $\$$}

Distributed under a Creative Commons Attribution - NonCommercial - NoDerivatives| 4.0 


\title{
NOTES
}

\section{LE FOURREAU D'ÉPÉE CELTIQUE DÉCORÉ DE BARON-SUR-ODON (Calvados)}

\author{
par Dominique BERTIN
}

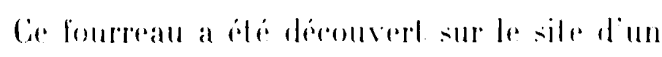
sancluaire gallo-romain, qui reenemse probablement un lien de rulle rellique. ou du moins un habilat gaubois. et qui ful partiellemenl fouillé par des amaleurs en 1951. puis par nous depuis 1969.

le monument appartient a la ralegorie des sancluates rello-romains, mais il est d'un lype un pera sperial : deagonal. il est miquerment ronstilue de deux galeries ronerent riques destinese an rite de la deambulation: il ne semble pas aroir de cella. Construil en male-

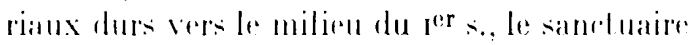
a subi divers remaniements jusqu'au milien

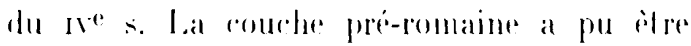
scindee en deux niveaux : le premier contenant de la ereramique bien datable de la Teme linale. le sceond. plus profomel, la ceramigues

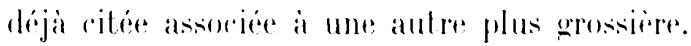
la straligraphere ne nous permet pas de faire remonter la datation de re nivean antedela du dibul de I.a Time 111.

Xous avons découvert le gourrean il y a douze ans, all rentre approximalif du momament, an sommet d'un las de déhlais provenanl de foulles anciennes. (1) pent done supposeser qüil proviend, d'un des niveans préromains du monument. Par suite de cireonstanees quil serait trop long de relater. lobjel est devenu la posisesion de .I. J. Menry, qui en a lait don al la l)irection des. Int iquités.

lat partic do fourrean ronservere a une longueur de 2fi.5 rom el une epaisseur maximale de 1,1 rom. En lien des endroils, la lame est a nu el les parties restantes du fourreau sont lres allaquées par l'oxydalion flig. 1: Ia radiographie n'a pas éle utile pour l'élude du décor. mais elle a permis de mieus distinguer lépere de son fourreaue el a ainsi facilite

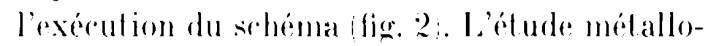
graphiques a mont ré que le mode de babrication de la lame romsiste en une juxlaposilion par soludage de lamelles de fer. suive d'un martelage a chand. I a boulerolle el la barrette transiersale onl disparu; on ne pent rien dire de la sole de lópee el le fourreau n'a pas l'aspect piqueló d'une piére travaillée selon la technique du rhagrinage. methode d'ornementalion employes surtoul a partir de

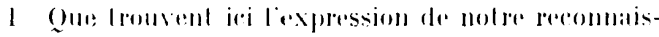

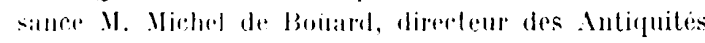
historignes de llaule ol Basse Normandie, gui nous a confir la publiation de a fomereall, ansi que

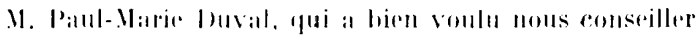
dians rer Iravial.

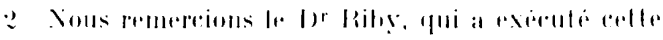
ranliographise.

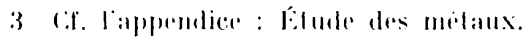




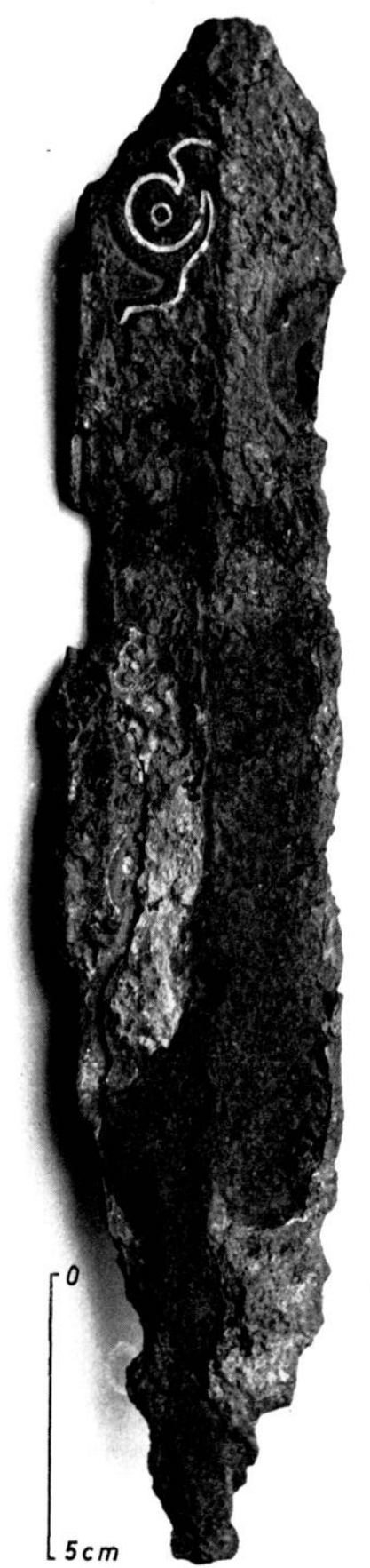

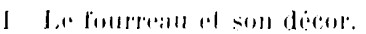

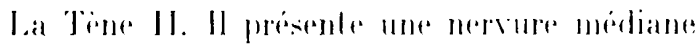
hien marduee el. sur le bord superieur gaurhe: on observe un lixer bourrelet romstitue sans doule par la pliure de la fendle de for inférienere sur la fouille superientur.

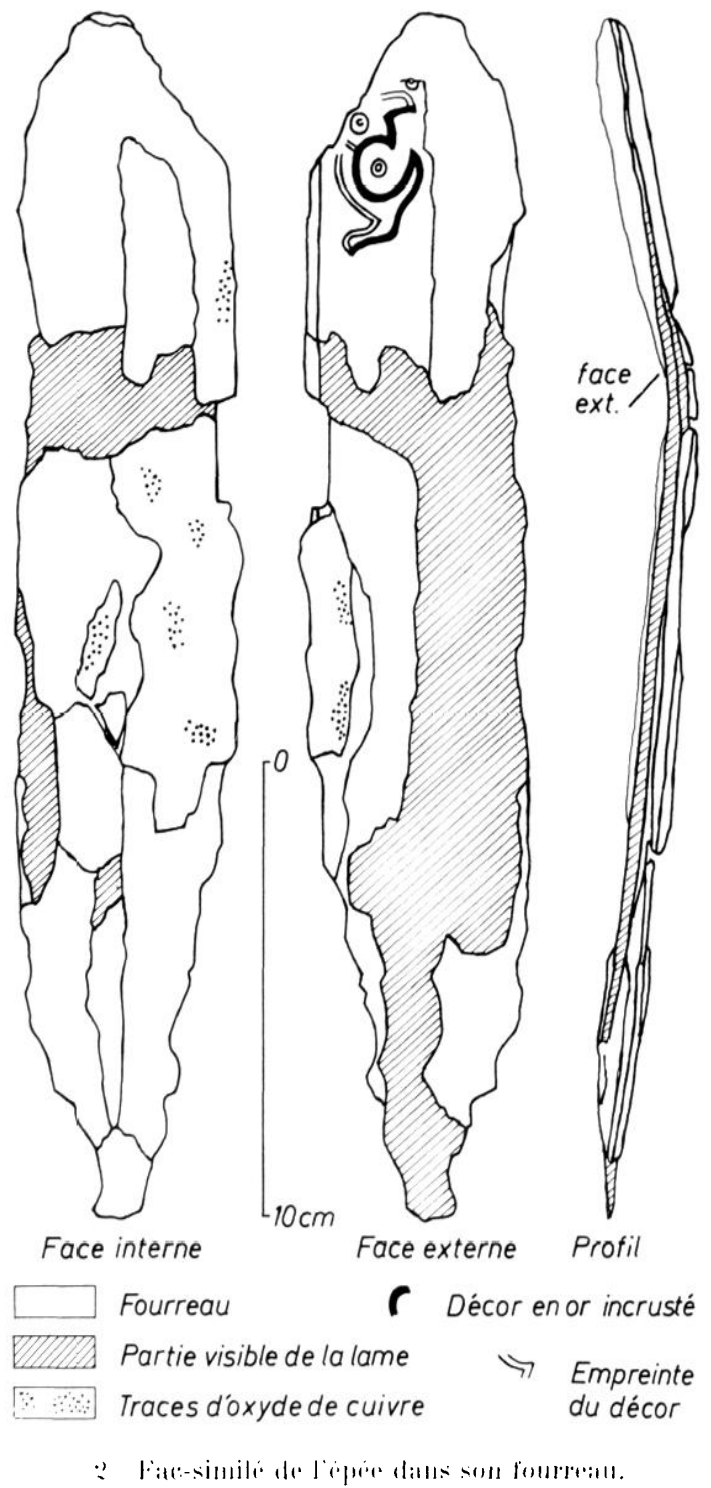

La romblume de louverture at assery pronomeres. sans loutefois ressembler vraiment. a tone reloche on a un chapeau de gemdarme. Elle est dissymétrique el presente un coté fresplue droit landis que l'aul re est franchement. enovese. Par ailleurs. elle se rétrécit fortement, vers le milien. sans loutefois se leminer an pointe ef elle esl assez haule (3 rmi; on pourrail done la rallacher au lype 12 d'ouverlures de fourreaux, détini récemment par

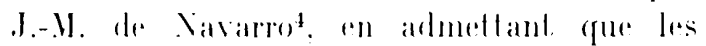

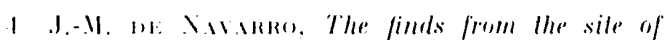

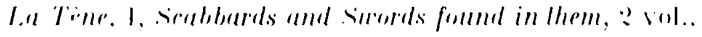
(1) ford, 197:?, p. :3. 
bords concaves raracteristiques de ce lype ont éte rassés, comme le laisse supposer le déror incomplet. I a plupart des fourreaux a dragons ont dialleurs des ouvertures du lype 12 . L'ouverture de notre fourreau affecte rependand une forme trop peu caractéristique pour itre un indice rhromologrique précis.

l'ensemble lane et fourreau a subi une legere pliure, peut-itre accidentelle, a 4 cm environ au-desones du décor. Enfin, nous avons remarque des traces verdatres sur les parties restantes du fourreall. Yous ne pensons pas qu'il s'agisse d'oxyde de ruire et qu'il faille en déduire que le fourreau ait ate doublé d'une feuille ou d'ornements de bronze, comme cest le cas pour quelques rares fourreaux de La Tène I. De plus, un revetement en bronze. du fourreat serait peu compatible aler le décor incruste dior encore existanl.

On aurail pus supposer, en raison des faibles dimensions de lobjet et de la forme eflilée de la rassure, que nous avions affaire non pas a une cepere, mais a une dague, du type des poignards courts que le chanoine P.-II. Favret considerait comme caractéristiques de la nécropole hallstatlienne des Jogases, ou du type de la dague de Weisskirchen, datee de Ia Tene Ia par P. Jarobsthat5. Nous ne pernsons pas qu'il faille ranger l'arme de Baron dans relte catégorie, car jamais il n'a ele trouve de fourreau d'épé rourte porlant un décor analogue. Ein outre, la stratigraphie du site de Baron nous interdit une dale ansis blevere que la Teine la.

Le décor est conservé sur un roté seulement de la nervure mediane, mais on peul affirmer aver une quasi-cerlitude qu'un lécor similaire lui faisail pendant, lequel a disparu par suite de la trop grande dégradation du fourreau : la paire de dragons opposés est en effet connue par un grand nombre de fourraux lalemiens fig. :3). La lechnique employee iri est celle de l'incrustation. sur la plaque de fer polie, les sillons da décor ont elé incises, puis remplis par un fil d'or. Iees sillons sont de section reclangulaire, ils sont larees d'environ $1,1 \mathrm{~mm}$ at profonds de $19,9 \mathrm{~mm}$ : leur fond est uni.

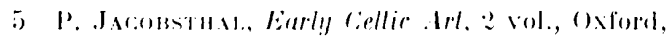

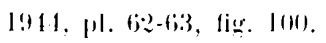

alors que la technique de l'incrustation laisse voir généralement les stries sur lesquelles adhere le métal. Ie fourreau de Baron est le: seul fourreau qui, a notre connaisisance, possede une incrustation dor ${ }^{b}$. Le fourreau d'Ering (Iaute-Baviere; oftre une incrustation, sans doute de corail, et peul-etre dautres fourreaux presentaient-ils aussi des incrustatlions.

Lo theme du décor est celui de la "paire de dragons", particulierement étudié par P. Jacobst hal en 194.47, et par J.-.11. de Navarro "n 19598 et en 19729. Ce demier savant a recensé en Europe to fourreaux offrant un décor de ce lype on de type apparente, figurant soil une paire de dragons, soit une paire d'«oiseaux", - les deux motils etant souvenl difliciles a distinguer f parfois associée a un motif complementaire. Neuf de res fourreaux proviennent du sile meme de La Tene. six de Suisse, l rois d'Allemagne, quatre de France, dix de Hongrie, deux de Yougoslavie, qualre de Trhécoslovaquie, un de Pologne, trois de la région intermédiaire entre la zone celtique de l'ouest el la zone cellique de l'Est, ... Haute Bavire et Basse Autriche.

Les deux dragons se fonl face de part el d'autre de la nervure médiane du fourreau qui, selon .J.-Y. de Navarro, est un trait d'archaisme; Il me Bret\%-Mahler considere que cette parlicularite qui, du point de vue lechnique, renforce le fourreau, napparait, que sur des fourreaux remarquables, en particulier par leur ornementation. I.es fourreaux du rimetirere de Sante- Memmie (Marne), datés de la Tiene Ir, sont nervures et la nervure médiane apparait aussi a La Tene Ic sur le fourreau a dragons de Münsingen (lig. 3). A notre comnaissance, la nervure médiane apparail sur tous les fourreaux a lécor zoumorphe; telle semble faire corps area re lype d'ormementation, puisque le déror sorganise de part al d'aulre de cette ligne.

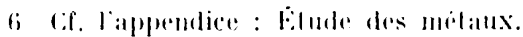

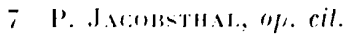

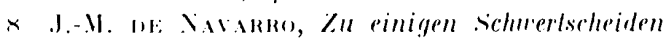
ans la Tine, daus do" Berichl der rämisch-germanischen Kommission, 1959, p. 7!-118, :23 pl.

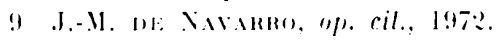




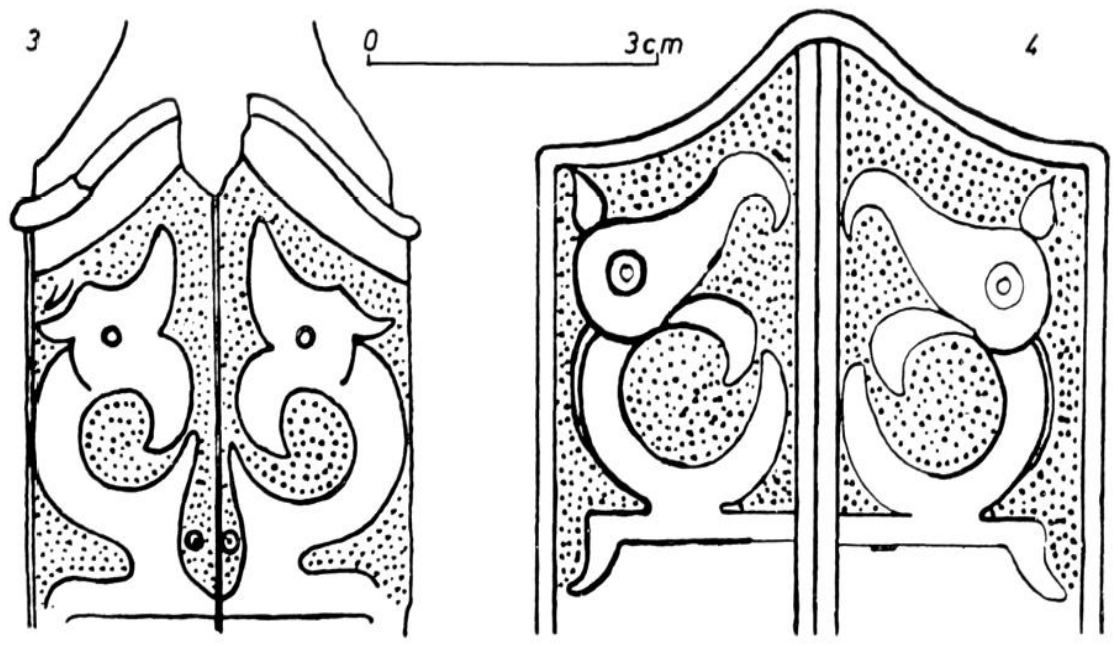

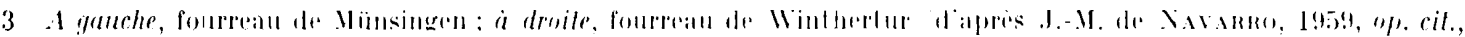

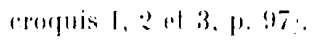

Sur le fourreau de Baron. le dragon de waurhe subsiste seul. legerement delériori an sommmel du cràne. Il a la guevile beante el recourbese: on distingue nellement son ail rirrulate str le bord superieur caluche du fourrean; sil avait une oreille. elle a ete emporter par la cassure. Le corpes de petite laille par rapport $\therefore$ la grueule. est arrondi, et un appendice remonte en avant jusquáa la bauteur de la partie inférieure de la gueule, sans la loucher. Dans le bas du corps se trouve une patte en forme de crochel, lournée vers larritre. Devant le corps du dragon se trouve un cercle? ocule al un aulere se distingue all-dessus de la marhoire superiesure; ces cereles incises completent to theme principal. Mise a part celte derniere particularite; le dragen est absolument conforme an lype l des dragons étudiés par .J.-M. de Navarro, lype qui est. a son avis le plus precoce (lig. 3).

I)ans le type III. l'appendice anlérieur rat ratlarhe a la marhoire inferieure. et l'ensemble forme un rerele ferme; on trouve ce lype de dragons surtout en suisse al au sile de la Tene. Quant au lype II, il est constitur par deux dragons dont le corps est en S, observant une courbe exactement inverse de celle des dragons du lype I, el l'ensemble des deux dragons évoque le motif de la lyre. Enlin. le corps de ces dragons it lermine par uno spirale.
J.-.ll. de Xavarro a rencontré neul lovis les dragens du lyper I sur des fourreaux deperes rell iques : qualere fois dans la zone celt ique de

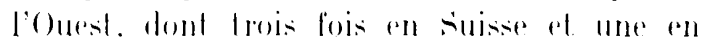
Frances. a Cihalons-sur-llarne ${ }^{10}$; qualere fois dans la zone reeltique de l'Est. dont frobs fors en llongries et une en Bohèment dans la zone

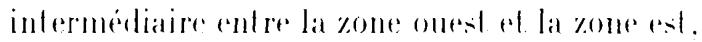
une fois en basse Autriche ${ }^{22}$.

Cese fourreatux proviennent de sepullures dans sept ads, et dans deux ras ils onl ele Irouve par dragage. Le fourrean de Vünsingen es date de Ia Tene Ie, el les aul res fourralux sont dates de la période de lamsition entre la Tene I el la Tene Il. Vous pensons done pouvoir dater le fourreau depée de Baron de la période de transilion entre la Tiene I el la teme I I, ou du lout débul de la l'ine II. Lne difficullé se live alors, aar les couches les plus profondes du site de Baron étudiés actuellement sont datables du début de lat Tine III.

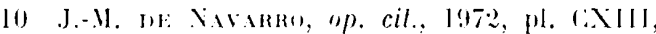

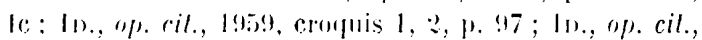
1959. cerpuis 1, 3. 1. 97; I11., op. rit., 1972. pl. СXJ.I, 11.

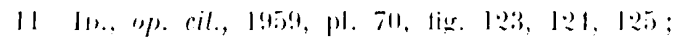

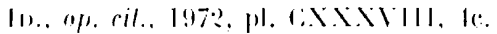

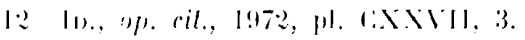


Touldefois, rommere la similitude du deror le

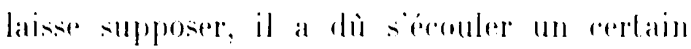
lape de lempe entere le moment oi le lourreaus découvere a baron a ele fabrique dans un

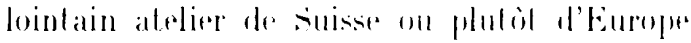
de l'est, el le moment oil il as arrive en Cormandie. Ein outere. an objel de luxe tel

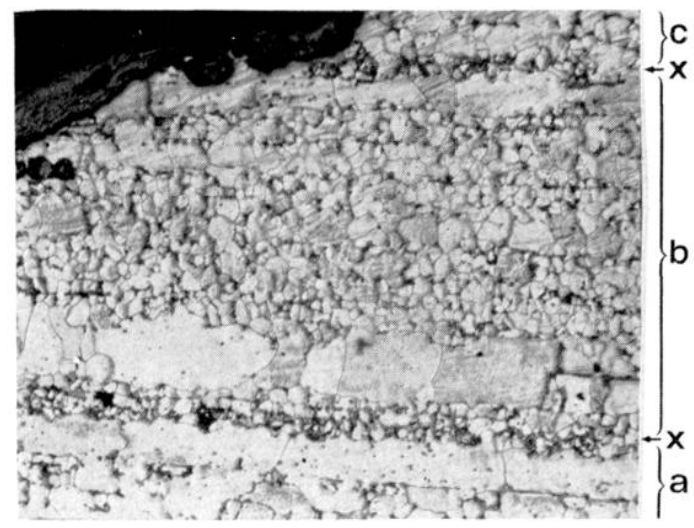

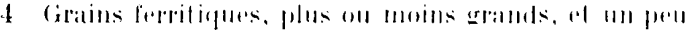

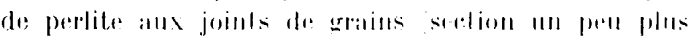

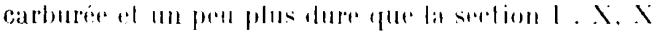

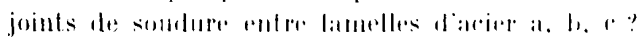

\section{IPPENTIER:}

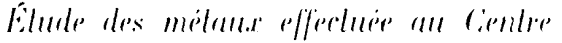

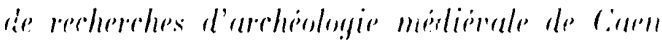

1. Loblude medallographigue al rhimigute a porté sur demx predievements eflecelues dams

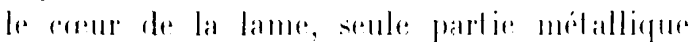

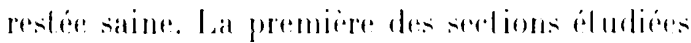
présente de lareges grains de ferrite avere quelques inclusions de serories: rest lun for

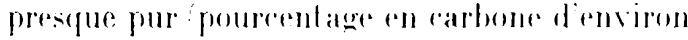

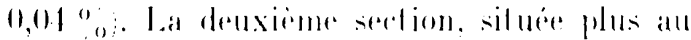
cour de la lame. presente une certame irrogularile dans la grosseren des grains de leprite. el montro la presence de perlile anx joints de ces grains fige 4. Celle structure caracterise

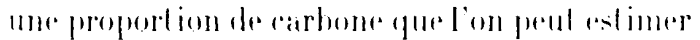

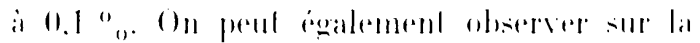
photographe de celle section des plages bien delimites vaisomblablement allribuables a

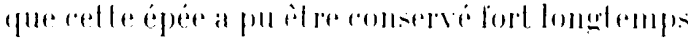
a transmis de generations en generalions.

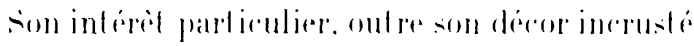

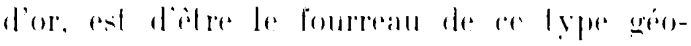

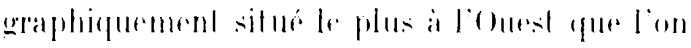
ail derentarert justua present.

Hominiqur Brats.

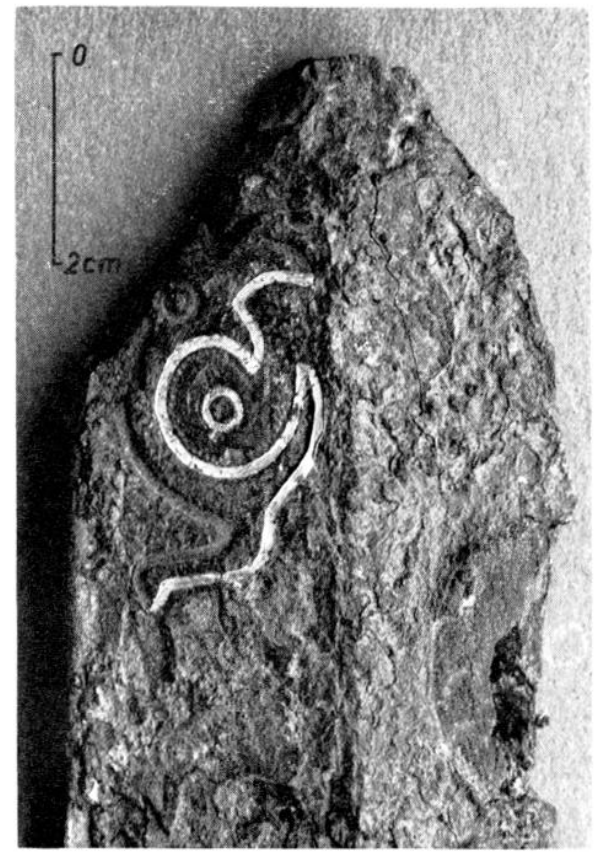

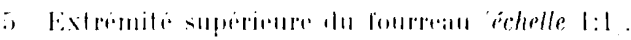

mene juxlapusition par soludage des lamelles de lere. Enlin. Ia morpholongie des grains de ferrile montre qu'il n'y a pas en de marlelage a froid an nivean des serelions eludieres.

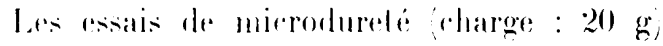
ellectures sur les deux sections ont foumb les resullals suivants: $1.10 \mathrm{~kg} / \mathrm{mon}^{2}$ pour la forrile de la sertion $1 ; 100 \mathrm{~kg} / \mathrm{rm}^{2}$ pour la ferrile de:

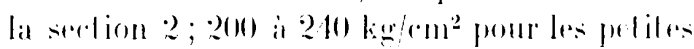

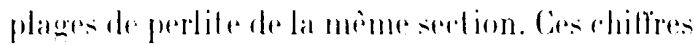
monlerent que le rerour de la lame n'a subi

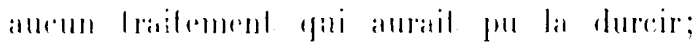

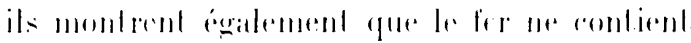

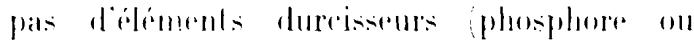
nitrures en particulier, " ce qu'un axamen 
métallographicque a plus fort grossissement nous a par ailleurs permis de verifier.

L'analyse rhimique des deux prelevements a donné les résultals suivants :

silire : inférieur ou egal à $0,4 " 0$ phosphore : muiron 0,5 ")"

manganese: present en quanlité non négligeable

lilane : inférieur a 0,$0 ; \%$ \% ou absent.

La forte leneur en phosphore ne correspond absolument pas aux duretes mesurées el ceri provient tris vadisemblablement d'une areumulation superficielle de cel clement dans la couche oxydere.

Si les résultats oblenus peuvent a la rigueur laisser penser que l'àme métallique de l'épée est constituée de fer presque pur. aurune donnée n'en permel une inlerpretalion plus grobale. Il reste toulefois quil est asse\% courant que le cour des armes soil constilue de fer netlrement moins riche en carbone que la périphérie, el phus particulierement le tranchant, pour donner plus d'élasticité a la lame. Getle arme a pll subir des traitements lhermiques superficiels. outre la cémentation, destines a durcir le Iranchant (la trempe, par exemple, et que la localisation forcée de celle elude au reur de la lame ne peut permettre de délinir.

2. Elurle du melal incrusté (fig. o) : leatal de conservation, la couleur el le diagramme de diffraction des rayons $X$ permettent d'aftirmer que le métal de base du décor est l'or. La présence d'argent en quanlite non négligeable a pu en outre ilre décelé ehimiquement. l'autre part, cerlaines observations effectuces sur le spectre de diffraction ronfirmeraient la présence d'un ément lel que le cuivre, couramment rencontré comme impurele dans l'or; dans l'affirmalive, les ralculs permell raient d'attribuer à cel elément une proportion pondérale d'environ $0,70 !$

Daniel Derockiner. 